\title{
-NOTES-
}

\section{ACCURATE APPROXIMATIONS TO THE POLYGAMMA FUNCTIONS}

\author{
By C. STUART KELLEY (U.S. Army Electronics Research \& Development Command, \\ Adelphi, Maryland)
}

\begin{abstract}
Empirically obtained approximations to the polygamma functions $\psi^{(n)}(x)$ and their associated accuracies are presented. These simple functions are quite accurate, this accuracy increasing with $x$ and being best for small $n$. These approximate expressions are shown to be circuitously related to the Stirling approximation to the parent gamma function.
\end{abstract}

1. Introduction. The absorption and emission spectral lines originating with free ions are considerably broadened when these ions are inserted as impurities in solids. This broadening is due to vibrational effects, where, for each electronic transition, there can be many exchanges of vibrational quanta (phonons) from the lattice or from local vibrational modes. At very low temperatures, only vibrational emission is possible-not absorption. [1-3] are samples of the work in this field.

At low temperatures the intensity $I$ of the absorption spectrum for such vibrationallyassisted transitions can be accurately described [2] by the Poisson shape

$$
I(E)=e^{-s} S^{(E / \hbar \omega)} / \Gamma(E / \hbar \omega+1) .
$$

The phonon energy is $\hbar \omega$ and $E$ is the photon, or optical, energy (relative to some reference) at which the absorption intensity is observed. The Huang-Rhys factor, $S$, is a measure of the strength of the vibrational interaction. Typical band shapes are shown in Fig. 1. The band shape, Eq. (1), appropriate to raising the ion from its electronic ground state to its electronic excited state can be reflected in a mirror-image fashion about $E=0$ to describe the emission spectrum that corresponds to an electronic transition from the ion's excited state to its ground state. $\Gamma(x)$ is the gamma function

$$
\Gamma(x)=\int_{0}^{\infty} t^{x-1} e^{-t} d t, \quad \operatorname{Re} x>0 .
$$

The peaks of the spectra occur when $d I(E) / d E=0$ at the value of $x=x_{p}$, where $x=$ $E / \hbar \omega$. Taking the derivative of Eq. (1), one finds

$$
\psi\left(x_{p}+1\right)=\ln S,
$$

where $\psi$, the psi or digamma function, is defined by

$$
\psi(x)=d[\ln \Gamma(x)] / d x .
$$

There are no closed-form expressions for $\psi$, but it can be evaluated [4] by series expansions: (a) when $x$ is an integer, (b) when $|x|<2$, and (c) asymptotically. Asymptotically,

$$
\psi(x) \sim \ln x-1 / 2 x-\sum_{k=1}^{\infty} \frac{B_{2 k}}{2 k x^{2 k}},
$$

where the $B_{n}$ are the Bernoulli numbers [4]. 


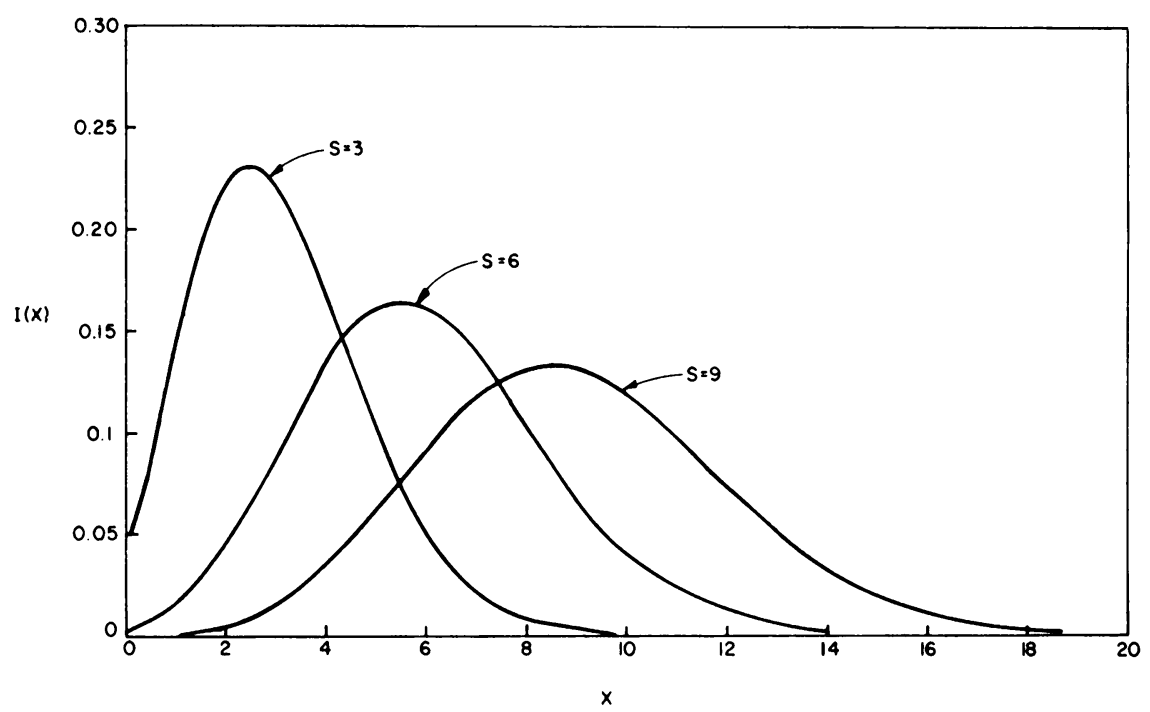

FIG 1. Poisson shapes, Eq. (1), representative of absorption spectra of impurities in solids parameterized by $S$, the Huang-Rhys factor.

There are available no simple approximations to $\psi(x)$ nor to its derivatives $\psi^{(n)}(x)$, the polygamma functions. However, some observations by solid-state physicists concerning the energy of the peak positions of absorption and emission spectra are relevant to the development of accurate approximations to $\psi$ and $\psi^{(n)}$.

2. Observations, approximations and accuracies. It has been observed $[1,2]$ that the Poisson line shape, Eq. (1), peaks near $x_{p} \simeq S$, or, better, that it peaks [5] near

$$
x_{p} \simeq S-\frac{1}{2} \text {. }
$$

In Table 1 we compare values of $S-1 / 2$ with values of $x_{p}$ found using Eq. (3) for a variety of values of $S$. As may be seen from the table, Eq. (6) is quite accurate, and increases in accuracy as $x$ increases. Thus, on this empirical basis, Eq. (3) is well approximated by

$$
\psi\left(x_{p}+1\right) \simeq \ln \left(x_{p}+1 / 2\right)
$$

for $x_{p} \sim 1$, and becomes increasingly accurate as $x_{p}$ increases.

TABLE 1. Values of $x_{p}$ for various values of $S$, the Huang-Rhys factor. These values of $x_{p}$ are closely approximated by the corresponding values of $S-1 / 2$, the approximation improving with increasing values of $S$.

\begin{tabular}{rcc}
$x_{p}$ & & $S-1 / 2$ \\
\cline { 1 - 1 } 0 & & 0.062 \\
1 & & 1.026 \\
2 & & 2.016 \\
3 & & 3.012 \\
4 & & 4.009 \\
5 & & 5.008 \\
6 & & 6.006 \\
50 & & 50.0008
\end{tabular}


The location of the peak of the absorption spectrum, Eq. (1), can also be estimated by noting that Eq. (1) can be rewritten as

$$
I(x)=I(x-1)(S / x) .
$$

Notice that $I(x)=I(x-1)$ when $x=S$. Thus the peak of the spectrum is bounded by

$$
S-1<x_{p}<S .
$$

As $S$ increases, the coefficient of skewness (here [6], $S^{-1 / 2}$ ) of the absorption spectrum decreases and the absorption spectrum becomes increasingly symmetric. For this reason, $x_{p} \rightarrow S-1 / 2$ as $S$ increases. Because the absorption spectrum is always skewed to the high-energy side (the coefficient of skewness is always positive), $x_{p}$ is always less than $S-$ $1 / 2$. Then

$$
S-1<x_{p} \leq S-1 / 2 .
$$

The accuracy of Eq. (7) suggests that the polygamma functions

$$
\psi^{(n)}(x) \equiv d^{n+1}[\ln \Gamma(x)] / d x^{n+1}, \quad n=1,2,3, \cdots
$$

may also be well approximated by the derivatives of Eq. (7),

$$
\psi^{(n)}(x) \simeq(-1)^{n-1}(n-1) \cdot\left(x-\frac{1}{2}\right)^{-n}, \quad n \geq 1 .
$$

We define the fractional errors in $\psi(x)$ and $\psi^{(n)}(x)$

$$
\begin{gathered}
\epsilon_{0} \equiv\left[\psi(x)-\ln \left(x-\frac{1}{2}\right)\right] / \psi(x), \\
\epsilon_{n} \equiv\left[\psi^{(n)}(x)-(-1)^{n-1}(n-1) \cdot\left(x-\frac{1}{2}\right)^{-n}\right] / \psi^{(n)}(x), \quad n \geq 1
\end{gathered}
$$

as measures of the accuracy of the approximations, Eqs. (7) and (12). Fig. 2 shows the dependencies on $x$ of $\epsilon_{0}$ through $\epsilon_{3}$. As can be seen from that figure, the approximations of Eqs. (7) and (12) all become more accurate as $x$ increases. Eq. (12) becomes less accurate as $n$ increases.

3. Discussion. Although we have considered only $\psi^{(n)}(x)$ for positive $x, \psi^{(n)}$ $(-x)$ may be determined through use of the reflection formula [4]

$$
\psi^{(n)}(1-x)=(-1)^{n}\left[\psi^{(n)}(x)+\pi d^{n} \cot (\pi x) / d x^{n}\right]
$$

and the recurrence formula [4]

$$
\psi^{(n)}(x+1)=\psi^{(n)}(x)+(-1)^{n} n ! x^{-n-1} .
$$

In the asymptotic formulas, Eq. (5) and [4]

$$
\psi^{(n)}(x) \sim(-1)^{n-1}\left[(n-1) ! / x^{n}+n ! / 2 x^{n+1}+\sum_{k=1}^{\infty} B_{2 k}(2 k+n-1) ! /(2 k) ! x^{2 k+n}\right],
$$

the leading terms differ from the approximations, Eqs. (7) and (12), only by the presence of $x-1 / 2$ in Eqs. (7) and (12) as opposed to $x$ in Eqs. (5) and (16). The use of only the leading terms in Eqs. (5) and (16) introduces values of $\epsilon_{n}$ that are about 300 times larger than those found using Eqs. (7) and (12), even for $x$ as large as 50. 


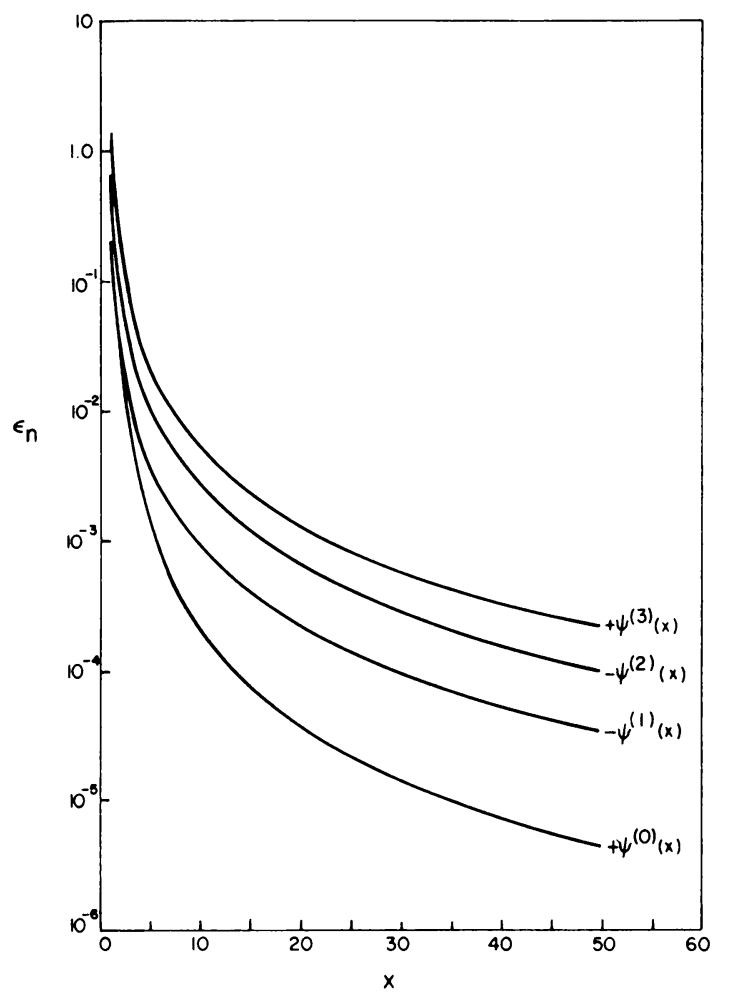

FIG 2. Dependence on $x$ of the fractional errors $\epsilon_{n}$ in $\psi^{n \prime}(x)$ that are incurred by the approximations of Eqs. (7) and (12).

If we approximate $\psi^{(n)}(x)$ by the derivatives of the asymptotic formula for the gamma function [4]

$$
\ln \Gamma(x) \sim\left(x-\frac{1}{2}\right) \ln x-x+\frac{1}{2} \ln (2 \pi)+\sum_{k=1}^{\infty} \frac{B_{2 k}}{2 k(2 k-1) x^{2 k-1}}
$$

when the $k$-sum is neglected (i.e., the Stirling approximation), we incur errors that are about twice those incurred when Eqs. (7) and (12) are used.

4. Concluding Remarks. The Stirling approximation (the three leading terms of Eq. (17) or the two leading terms of Eq. (5)) can be shown to be related to the empiricallyobtained approximation to $\psi(x)$, Eq. (7). Applying the definition of $\psi(x)$, Eq. (4), to the three leading terms of Eq. (17), we obtain the two leading terms of Eq. (5), which may be rewritten as

$$
\psi(x) \simeq \ln \left(x \exp \left(-\frac{1}{2 x}\right)\right)
$$

Expanding the exponential and retaining the first two terms gives

$$
\psi(x) \simeq \ln [x(1-1 / 2 x)]=\ln \left(x-\frac{1}{2}\right),
$$

which is just the empirically observed approximation, Eq. (7). 
In conclusion, we find that the empirically-obtained approximations, Eqs. (7) and (12), to the polygamma functions $\psi^{(n)}(x)$ are quite accurate, especially for large $x$ and/or small $n$.

\section{REFERENCES}

[1] J. J. Markham, Rev. Mod. Phys. 31, 956 (1959)

[2] T. H. Keil, Phys. Rev. A 140, 601 (1965)

[3] C. S. Kelley, Phys. Rev. B 6, 4112 (1972)

[4] Handbook of mathematical functions, ed. M. Abramowitz and I. A. Stegun, Natl. Bur. Std. (U.S.), Appl. Math. Ser. (U. S. Government Printing Office, Washington, D.C., 1964; Dover, New York, 1965); see especially chapter 6 .

[5] C. S. Kelley, Phys. Rev. B 8, 1806 (1973)

[6] C. S. Kelley, J. Chem. Phys. 68, 1322 (1978) 